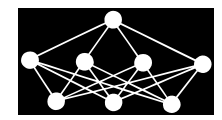

\title{
PHYSICS-INFORMATION ANALOGIES
}

\author{
M. Svítek*
}

\begin{abstract}
The paper presents a theory of information systems based on advanced analogies with both classical and quantum physical models. In the first step the information analogies with magneto-electric circuits are introduced and the information parameters are defined under this inspiration. Well-known potential and flow values (e.g. potential and kinetic energy, voltage and electrical current, etc.) are transformed into information values, "information content" and "information flow". In the second step the quantum information models are introduced together with values "wave information flow" and "wave information content". By using these variables, the complex information models are described in more detail together with illustrative examples.
\end{abstract}

Key words: physics-information analogies, information flow, information content, information gate, information dynamics, quantum information, quantum event, quantum gate, quantum system, information perception, smart city, transport telematic

Received: February 9, 2018

DOI: $10.14311 /$ NNW.2018.28.030

Revised and accepted: December 30, 2018

\section{Introduction in information epistemology}

A basic information unit represents a "change of state/signal" from 0 to 1 , or vice versa. The change is equal to one-bit of information. Information flow [15] means the frequency of state/signal changes in bits per second or how often the change is carried out (quantity of information). Information content [14], on the contrary, characterizes the quality of information or how valuable the content is, measured in Joule per bit (or dollars per bit).

In [22] the extended Frege's concept of information modelling was presented based on results $[9,14]$. Basic information quantities were given:

- $\mathbf{O}_{i}(t)$ - a set of rated quantities on an object,

$-\mathbf{P}_{i}(t)-$ a set of states,

- $\boldsymbol{\Phi}_{i}(t)$ - a set of syntactic strings (data flow),

- $\mathbf{I}_{i}(t)$ - a set of information images of state quantities (information content).

*Miroslav Svítek; Czech Technical University in Prague, Faculty of Transportation Sciences, Konviktská 20, Prague 1, Czech Republic, E-mail: svitek@fd.cvut.cz and Matej Bel University, Faculty of Natural Sciences, Tajovského 40, Bánská Bystrica, Slovak Republic 
The Frege's diagram can be also used to describe the deep perception of information by humans [25]. Psychologists accepted that it is possible to study the world around us from four points of views: physical, emotional, rational and spiritual.

Physical representation corresponds to changes of a real system (events $\mathbf{O}_{i}$ ). The human mind categorizes a perception into different clusters called states $\mathbf{P}_{i}$ which cover a mixture of intellectual and emotional stimuli assigned to a specific situation that occurred in a real system. The packet of such stimuli specifies the state of our perception that we try to characterize by words through thinking (generally by symbols according to the grammar).

Within this process the string of symbols $\boldsymbol{\Phi}_{i}$ is produced to best capture the perception of the state $\mathbf{P}_{i}$. Thinking has limited (often sequential) instruments for expression of the complexity of the world but it tries to provide an answer to the question "how"? Thinking is a conscious activity that can be unlike other conscious activities shared, written or published and it is a good background for further understanding of the whole.

The higher (spiritual) level means the link to the whole (higher order) and to other available knowledge like comparisons with similar situations in history and with knowledge from the other specializations, etc. This part of cognition looks for an answer to the question "why" the system behaves like that? It can be called wisdom, which is a higher level of knowledge stated generally as the spiritual level of the perception.

Let us give one easy example of music; an orchestra playing some composition. In the physical world we can see a lot of instruments that create sounds of different frequencies $\left(\mathbf{O}_{i}\right)$. If the sound is coordinated, we can extract some feeling and emotions as a state of our mood $\left(\mathbf{P}_{i}\right)$ that the music placed us in. Intellectual analyze can describe our mood by words $\left(\boldsymbol{\Phi}_{i}\right)$ and think about links to a higher order $\left(\mathbf{I}_{i}\right)$ - i.e. what the author wanted to say if he composed this song, etc. Understanding of all of the details yields to a better perception of the details of the song if we listen to it once again. A better perception a better understanding more details. The perception process could be repeated until a stable knowledge is accumulated and the song is understood to a given distinguished level.

We can go deeper and suppose that due to our model we can expect new features of reality and our perception can aim to this direction. Such a function could be called the insight sensor, which can direct us to correct details based on accumulated information. The more accumulated information the higher details we can register.

From the system point of view, we can not only go into more deeper layers, but we can extract an archetypal knowledge that is given as the stable state of continual information observation, perception, processing and creation of the appropriate model of reality on a given distinguishing level. Archetypal knowledge represents a clearly defined piece of knowledge that can be understood as the base component from which complexity can be composed. It is a new way for the description of a reality rather than the creation of a more and more complex high dimensional model.

The goal behind this discussion is to have available a set of simple archetypal models carrying extracted bases of knowledge. For example, in psychology we can go deeper and deeper in describing a person's behavior. But there are other 
possible ways to specify archetypal psychological personalities and suppose that the psychological profile of everyone is weighted with a mixture of these archetypal models. Each specialization can be tackled in this way. Instead of building a complex model the archetypal knowledge could be extracted, and the diversity can be studied through an archetypal knowledge composition.

Archetypal knowledge in music, for example, means the extraction of different moods represented by a set of features of the song. All other songs could be a mixture of archetypal songs together with the different moods they produce. Similarly this applies to architecture, religion, etc.

We can continue with our description of epistemology and suppose that there is only one reality $\mathbf{O}_{i}$ studied by many specialists. Each of them has their own insight sensor registering different details. If someone has their insight sensor working on a given level of resolution the expert has a chance to understand themselves and share their information $\boldsymbol{\Phi}_{i}$.

String of symbols $\boldsymbol{\Phi}_{i}$ is the only instrument we have at our disposal to communicate and to express our accumulated knowledge. It is necessary to understand both the syntactical part (it is easier task) and the semantical content that requires the insight sensor to be working on a given distinguishing level.

The sharing of information among experts extends their horizons and yields to combinations of knowledge from different areas (sharing archetypal knowledge). The characteristic called exaptation ${ }^{1}$ could be achieved, which means to make use of the knowledge from different specializations to make progress in our area of interest.

Let us imagine that we study a city as a complex system [12] and an architect understands the city from the urbanist point of view and looks at the urbanist details (in his perception the set of urban archetypal models exists), a sociologist uses its insight sensor and observes a lot of details about population, a business specialist looks at the market potential, etc.

Every expert can create his Frege's diagram [22] of the reality (knowledge component), which means to identify different scenarios $\mathbf{P}_{i}$ in its specialization as the optimized simplification of observed (cutout) reality $\mathbf{O}_{i}$.

With respect to the identified scenarios $\mathbf{P}_{i}$ the clear understanding (rational description) $\boldsymbol{\Phi}_{i}$ is available and each scenario should be included into the context [1] of higher knowledge $\mathbf{I}_{i}$. The multi-dimensional Frege's diagram assigned to different scenarios can be constructed. All Frege's diagrams are connected through the reality $\mathbf{O}_{i}$, which is of course unique for all scenarios.

\section{Information circuits}

\subsection{Classical information event}

Let us suppose that information is clustered into a higher semantic structure like our language. For simplicity, we can imagine a set of different events $u_{i}$ each of them is generally described by a syntactic chain e.g. $\{0,1,1,0\}$ of different lengths.

\footnotetext{
${ }^{1}$ For example, the analogy between electric, magnetic and informatics was used to make progress in complex system theory.
} 
In practical terms, one event can mean e.g. a recommended scenario of future behavior.

In such a case we have an information signal of a syntactic string of events $\left\{u_{1}, u_{2}, u_{3}, \ldots\right\}$ encoded into information flow of $\{0,1\}$. The value of the event is hardly the same, so we must count with different qualities assigned to each event. The scenario of the critical situation is more valuable than the scenario for a normal situation. Some events carry a low content, some a bit higher.

In the theory of information analogies, the link between the information source and the recipient must be better analyzed and generalized for the purposes of complex behaviors. On the side of the source, each event $u$ can be described by source information content and flow $\mathbf{I}_{S}(u), \boldsymbol{\Phi}_{S}(u)$. The recipient tries to process the event $u$ in its environment, provides its registration and its understanding. This process results in the representation of received information content and flow $\mathbf{I}_{R}(u), \boldsymbol{\Phi}_{R}(u)$ on the side of the recipient.

For a lot of events $u$ we can suppose that there is no difference between the information source and the received information. We rightfully suppose that:

$$
\begin{gathered}
\mathbf{I}_{S}(u)=\mathbf{I}_{R}(u), \\
\boldsymbol{\Phi}_{S}(u)=\boldsymbol{\Phi}_{R}(u) .
\end{gathered}
$$

For more complex events this assumption is not valid. In social sciences, for example, we must have a lot of data available (information flow) to identify some social event (information content). The link between the events originated in society and registered by observers having a dynamic time evolution.

\subsection{Quantum information event}

An occurrence of one event $u=1$ can be represented in quantum informatics by a unique wave function as:

$$
\psi(u=1)=1 \cdot \mathrm{e}^{j \cdot \varphi_{1,1}} .
$$

And the non-occurrence of the event $u=0$ by a different wave function:

$$
\psi(u=0)=1 \cdot \mathrm{e}^{j \cdot \varphi_{1,0}}
$$

where numbers 1 or 0 means that the event $u$ has happened or not happed and a phase $\varphi$ describes how the environment accepts the event $u$.

The phase difference can be caused either by measurement equipment such as quantum mechanics, or by an error of the human observer (he/she has glasses and badly registers the trials), or by natural resistance against the event (the receiving system does not freely accept the event $u$ ) $[17,18]$. These situations cause basic discrepancies between the reality (the event $u$ has happened) and the environment (the event $u$ is or is not registered by its environment).

Using this method of thinking we can add a phase parameter to each realization of unique event $u$. Generally, the phase can be different for each registered event because the environment can be time-varying, and the conditions cannot be the same in different time intervals.

If we conduct more trials in which the event $u$ happened (and did not happen) we can count the statistics as an average of these complex numbers. If all events are 
accepted in the same way we can compute the frequency of right trials (probabilistic description).

On the other hand, if each trial has different phase parameter this situation is reflected by a value of modulus and phase of the sum of wave representations and the wave probabilities could be computed as:

$$
\begin{aligned}
& \psi_{1}=\sqrt{p_{1}} \cdot \mathrm{e}^{j \cdot \varphi_{1}}, \\
& \psi_{0}=\sqrt{p_{0}} \cdot \mathrm{e}^{j \cdot \varphi_{0}} .
\end{aligned}
$$

The quantum system representation in Bracket or Dirac notation [24] can be written as:

$$
\psi=\psi_{0}|0\rangle+\psi_{1}|1\rangle .
$$

It tells us that even though we observed $m$ right fallings of separate events $u=1$, in summary, if we look on $N$ trials as the whole (not separately) we will observe less or more than $m$ right fallings due to phase interferences among trials. The Holistic view expresses a slightly different reality than the sum of individual observations $[16,19]$.

Let us present an illustrative example of five unique observations:

$$
\begin{aligned}
\psi \propto\left(\mathrm{e}^{j \cdot \varphi_{0,1}}|0\rangle_{1}\right. & \left.+\mathrm{e}^{j \cdot \varphi_{0,2}}|0\rangle_{2}\right)+\left(\mathrm{e}^{j \cdot \varphi_{1,1}}|1\rangle_{1}+\mathrm{e}^{j \cdot \varphi_{1,2}}|1\rangle_{2}+\mathrm{e}^{j \cdot \varphi_{1,3}}|1\rangle_{3}\right)= \\
& =\left(\mathrm{e}^{j \cdot \varphi_{0,1}}+\mathrm{e}^{j \cdot \varphi_{0,2}}\right)|0\rangle+\left(\mathrm{e}^{j \cdot \varphi_{1,1}}+\mathrm{e}^{j \cdot \varphi_{1,2}}+\mathrm{e}^{j \cdot \varphi_{1,3}}\right)|1\rangle= \\
& =\sqrt{\frac{2}{5}} \cdot \mathrm{e}^{j \cdot \varphi_{0}}|0\rangle+\sqrt{\frac{3}{5}} \cdot \mathrm{e}^{j \cdot \varphi_{1}}|1\rangle,
\end{aligned}
$$

where $\propto$ means equality up to the normalization factor. We can look at the combination of wave properties (wave probabilistic functions assigned to different states $|0\rangle,|1\rangle$ ) and at the corpuscular behavior (falling of different states $|0\rangle,|1\rangle$ ). This interpretation given for macroscopic systems is very close to "ghost waves" studied in quantum mechanics [2]. According to this theory, "ghost waves" continuously create the space of possibilities according to the quantum wave principles in which the particle behaves (falling one of the states) as a mechanical object.

Because we can register only the falling 0 or 1 (modulus of wave parameters) the phases could only be measurable based on statistics (an aggregation of the set of many realizations). Final phase parameters $\varphi_{0}, \varphi_{1}$ describe the dependence between statistics of falling 0 or 1 . Unfortunately, they do not carry a phase information about the unique phases $\varphi_{0,1}, \varphi_{0,2}, \varphi_{1,1}, \varphi_{1,2}, \varphi_{1,3}$. Such information is lost during an aggregation.

\subsection{Classical information gate}

The information sources can have the information content assign to the event $u$ but it cannot be presented because the event is very personal, secret, etc. Such a situation can be characterized:

$$
\mathbf{I}(u) \neq 0, \boldsymbol{\Phi}(u)=0 .
$$


There could exist ways of how to make such information partially visible e.g. through non-linear information processing ${ }^{2}$ that can cause the modulation of this event into other events.

On the other hand, the information source may not have any relevant information content, but it presents the event $u$ through the information flow (fake news). In such a case we use the following description:

$$
\mathbf{I}(u)=0, \boldsymbol{\Phi}(u) \neq 0 .
$$

There are other situations where any reasonable function between information flow and content cannot be found:

$$
\mathbf{I}(u) \neq 0, \boldsymbol{\Phi}(u) \neq 0, \mathbf{I}(u) \neq f(\boldsymbol{\Phi}(u)) .
$$

The information component with such a feature is called "information norator" [3].

On the other side, the "information nullator" guarantees zero values for every situations:

$$
\mathbf{I}(u)=0, \boldsymbol{\Phi}(u)=0 .
$$

Transmittance $R_{i}$ or conductance $G_{i}$ assigned to $i$-th component represents a linear dependence between information flow and content (analogy to well-known Ohm's Law) [22]:

$$
\boldsymbol{\Phi}(u)=\frac{\mathbf{I}(u)}{R_{i}}=\mathbf{I}(u) \cdot G_{i} .
$$

Other information components can include different information sources. Contentoriented information source means that information content is a constant independent of any requested information flow - it is typical for informatics. A Floworiented information source can be defined as a constant information flow even if the information content is varying.

For the sake of simplicity, let us imagine an information subsystem as an inputoutput information gate that issues from a matrix representation in the following form $[9,17]$ :

$$
\left(\begin{array}{c}
\mathbf{I}_{2} \\
\mathbf{\Phi}_{2}
\end{array}\right)=\left(\begin{array}{ll}
t_{a} & t_{b} \\
t_{c} & t_{d}
\end{array}\right) \cdot\left(\begin{array}{c}
\mathbf{I}_{1} \\
\mathbf{\Phi}_{1}
\end{array}\right)=\mathbf{T} \cdot\left(\begin{array}{c}
\mathbf{I}_{1} \\
\mathbf{\Phi}_{1}
\end{array}\right),
$$

where the matrix $\mathbf{T}$ is called the transmission matrix.

The basis of information systems is the ability to interconnect individual information subsystems, or in our case, input-output information gates. It is very easy to imagine the serial or parallel ordering of these subsystems into higher units. A very interesting model is feedback of information subsystems because this leads to non-linear characteristics, information systems defined at the limit of stability and other interesting properties.

\footnotetext{
${ }^{2}$ If you would like to know about this kind of information, you cannot ask for it. It is necessary to discuss the story in which this information appears. Emotions play a very important role.
} 
It is hard to find an appropriate system to combine the characteristics of the different information subsystems described above, but it is possible to create a group of subsystems - system alliance [26], where these characteristics can be combined appropriately.

\subsection{Quantum information gate}

Let us define the wave information flow and the wave information content [20] assigned to input-ouput information gate:

$$
\begin{gathered}
\psi_{\boldsymbol{\Phi}}=\alpha_{\boldsymbol{\Phi}, 1} \cdot\left|\boldsymbol{\Phi}_{1}\right\rangle+\alpha_{\boldsymbol{\Phi}, 2}, \cdot\left|\boldsymbol{\Phi}_{2}\right\rangle+\cdots+\alpha_{\boldsymbol{\Phi}, N} \cdot\left|\boldsymbol{\Phi}_{N}\right\rangle \\
\psi_{I}=\alpha_{\mathbf{I}, 1} \cdot\left|\mathbf{I}_{1}\right\rangle+\alpha_{\mathbf{I}, 2}\left|\mathbf{I}_{2}\right\rangle+\cdots+\alpha_{\mathbf{I}, N} \cdot\left|\mathbf{I}_{N}\right\rangle
\end{gathered}
$$

where $\boldsymbol{\Phi}_{1}, \ldots, \boldsymbol{\Phi}_{N}$ and $\mathbf{I}_{1}, \ldots, \mathbf{I}_{N}$ are possible values of information flow and information content ${ }^{3}$, respectively. Complex parameters $\alpha_{\boldsymbol{\Phi}, 1}, \ldots, \alpha_{\boldsymbol{\Phi}, N}$ and $\alpha_{\mathbf{I}, 1}, \ldots, \alpha_{\mathbf{I}, N}$ represent wave probabilities [20].

The information power can be expressed as follows [24]:

$$
\begin{array}{r}
\psi_{P I}=\psi_{\mathbf{\Phi}} \otimes \psi_{\mathbf{I}}=\alpha_{\boldsymbol{\Phi}, 1} \cdot \alpha_{\mathbf{I}, 1} \cdot\left|\boldsymbol{\Phi}_{1}, \mathbf{I}_{1}\right\rangle+\cdots+\alpha_{\boldsymbol{\Phi}, 1} \cdot \alpha_{\mathbf{I}, N} \cdot\left|\boldsymbol{\Phi}_{1}, \mathbf{I}_{N}\right\rangle+\ldots \\
\cdots+\alpha_{\boldsymbol{\Phi}, N} \cdot \alpha_{\mathbf{I}, 1} \cdot\left|\boldsymbol{\Phi}_{N}, \mathbf{I}_{N}\right\rangle+\cdots+\alpha_{\boldsymbol{\Phi}, N} \cdot \alpha_{\mathbf{I}, N} \cdot\left|\boldsymbol{\Phi}_{N}, \mathbf{I}_{N}\right\rangle
\end{array}
$$

where symbol $\otimes$ means Kronecker operation [5] for vectors transformed into multiplication, each $i, j$-th component $\left|\boldsymbol{\Phi}_{i}, \mathbf{I}_{j}\right\rangle$ represents a particular value of information power that characterizes the falling / measuring of the information flow $\boldsymbol{\Phi}_{i}$ and the information content $\mathbf{I}_{j}$.

Multiplication of different combinations of the information flows and contents $\left|\boldsymbol{\Phi}_{i}, \mathbf{I}_{j}\right\rangle,\left|\boldsymbol{\Phi}_{k}, \mathbf{I}_{l}\right\rangle$ can achieve the same information power $\mathbf{K}_{r}$ :

$$
\mathbf{\Phi}_{i} \cdot \mathbf{I}_{j} \approx \boldsymbol{\Phi}_{k} \cdot \mathbf{I}_{l} \approx \mathbf{K}_{r}
$$

Finally, an information power in renormalized form can be expressed as:

$$
\psi_{P I}=\beta_{1} \cdot\left|\mathbf{K}_{1}\right\rangle+\beta_{2} \cdot\left|\mathbf{K}_{2}\right\rangle+\cdots+\beta_{r}\left|\mathbf{K}_{r}\right\rangle+\ldots
$$

This approach yields to the resonance principle between the information flow and information content.

\section{Classical physics - information analogies}

\subsection{Electrics - information analogies}

In the current state of information analogies, an electric circuit with its electric current [coulomb per second] and voltage [Joule per coulomb] represents the analogy of an information model with quantities:

\footnotetext{
${ }^{3}$ Different variants of information flow and content mean the different point of view on the studied system.
} 
- Information content - I [Joule per bit] defines the model of a real system together with its appropriate features and suitable control strategies (extracted uncertainty of data flow, data interpretation, model structure identification, model parameters' estimation, mixture of multi-models, model's verification and validation and model-based control)

- Information flow - $\mathbf{\Phi}$ [bits per second] describes the syntax strings of data flow assigned to a real system (data collection and signals transmission to/from a real system).

The information model only works with available information and creates a suitable representation of a real system. In the electric analogy, Joule per bit means the energy required for finding the most suitable model for a real system.

\subsection{Magnetic - information analogies}

The magnetic circuit with the magnetic flux $\varphi$ [Joule-second per coulomb] and with the magnetomotive force (mmf) $F$ [Joule-second per coulomb] represents the action assigned to one coulomb. Action is an attribute of dynamics of a physical system from which the equation of real motion can be derived. In this paper we will rather use the "rate of magnetic flux" $\frac{\mathrm{d} \varphi}{\mathrm{d} t}$ in [Joule per coulomb] which is related to the energy flow (energy transmission) carried by a coulomb.

In information analogies it means the changes of a real system to achieve more energy. Such approach is like the Kauffman's principle of self-organized agents [8] continuously looking for more and more sophisticated ways of obtaining energy.

The electric - magnetic transformation can be modeled by a gyrator as it is shown in Fig. 1. On the left side there are electrical parameters: $v$ - electric voltage, $i$-electric current and on the right site there are magnetic parameters: $F$ - magneto motive force ( $\mathrm{mmf}$ ) and $\mathrm{d} \Phi / \mathrm{dt}$ - rate of magnetic flux ( $\Phi$ is not bold).
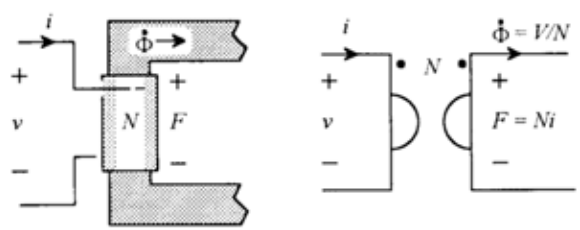

Fig. 1 Gyrator model of electric / magnetic transformer [7].

For a winding of $N$ turns we can write:

$$
\begin{gathered}
v=N \cdot \frac{\mathrm{d} \Phi}{\mathrm{d} t}, \\
i=\frac{F}{N} .
\end{gathered}
$$

The magnetic circuit (right side of Fig. 1) represents in an information analogy a modification of a physical system based on the available information (left side of Fig. 1). With respect to this it is possible to define two new information parameters 
assigned to a real physical system (to distinguish the right side of Fig. 1 we use the term 'knowledge-based'):

- Knowledge-based action A [Joule-second per bit] - Magneto motive force $(\mathrm{mmf})$ can be interpreted in magnetic-information analogy as an action that describes the amount of energy ${ }^{4}$ that could be obtained from a real system based on one-bit of information during one second. Different changes of a real system yield to different values of physical actions. Information flow $\mathbf{\Phi}$ from an information model identifies actions in the real world. On the other hand, new actions (in the real system) can generate new information flows or, in other words, a new modification of the information model.

- Knowledge-based energy flow $E$ - the rate of magnetic flux $\mathrm{d} \Phi / \mathrm{dt}$ in the magnetic-information analogy can be interpreted as knowledge-based resources extraction. The information content $\mathbf{I}$ represented by the information model enables us to realize changes of the real physical system (changes in structure, organization, processes, etc.) in such a way that new resources of identified energy could be extracted. Parameter $E$ describes the speed of flow of the extracted energy in [Joules per second].

\subsection{Illustrative example- smart city transport modelling}

We can imagine a transportation system in a closed urban area (smart city district) that must be controlled by coordinated strategies of traffic lights [23]. First, it is necessary to build a model of the urban area using historical traffic data. The model can explain the traffic behavior on a given resolution level. If we collect on-line traffic data from traffic detectors the model must decide which is the most appropriate scenario (traffic control strategy) to use. In our terminology it means that we have the information gate with input information $-\boldsymbol{\Phi}_{1}, \mathbf{I}_{1}$ given by collected on-line traffic data from the urban area $\boldsymbol{\Phi}_{1}$ and the historical knowledge $\mathbf{I}_{1}$.

The information gate processes the input data $\boldsymbol{\Phi}_{1}, \mathbf{I}_{1}$ and based on the traffic model, decides which control scenario $\mathbf{I}_{2}$ is the best one for current situation. According to the scenario the appropriate control signals $\boldsymbol{\Phi}_{2}$ are distributed to the traffic lights. Such an information gate is a typical example of the electrical analogy where we are working with the input / output data flows and the information contents as it is shown in Fig. 2.

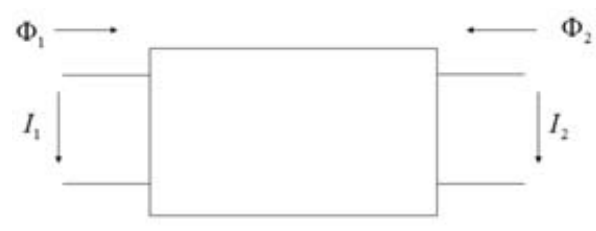

Fig. 2 Information gate - the control based on the selected scenario.

The values $\boldsymbol{\Phi}_{2}, \mathbf{I}_{2}$ are transformed into the real traffic system (analogy to electric / magnetic transformer) where they cause the knowledge-based actions $A_{1}$ (new

\footnotetext{
${ }^{4}$ The new source of energy that can be exploited using available information
} 
cars' flows). There are many possibilities, but we expected that the model $\mathbf{\Phi}_{2}, \mathbf{I}_{2}$ has recommended to us the best variant ${ }^{5}$ of how to optimize (Fig. 3) the saved energy flow $E_{1}$ (minimum air pollution, minimal cars' stops, maximal speed of traffic flow, etc.) of the urban area.

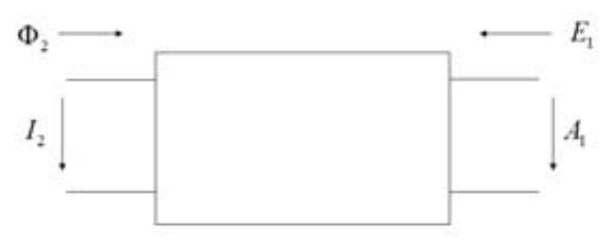

Fig. 3 The information transforms into a knowledge-based action and energy flow.

We noted that there could be some changes to the real system made without the information model. Local knowledge can be applied to react on some unexpected events. On the other hand, we can also expect a reduction of dimensionality to the studied system because of only a partial observation (cutoff). Such changes can also be modelled by the gate shown in Fig. 4 .

The model transforms the original values $E_{1}, A_{1}$ into the new ones $E_{2}, A_{2}$.

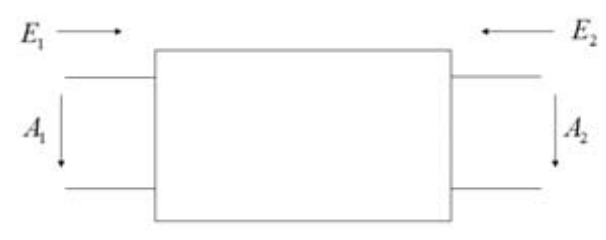

Fig. 4 The model of changes on the side of the real system.

Next, we observed the behavior of the traffic subsystem $E_{2}, A_{2}$. We identified new pieces of information that can be measured on the real physical system. For example, we have at our disposal the on-line GPS (Global Positioning System) with positions assigned to some vehicles ${ }^{6}$. By their processing as shown in Fig. 5 we obtain more detailed model of the physical system $\boldsymbol{\Phi}_{3}, \mathbf{I}_{3}$.

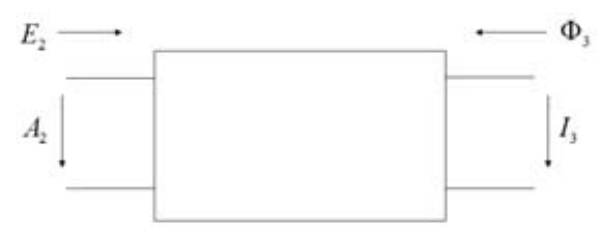

Fig. 5 The information extraction from the real system.

The extracted information (analogy to magnetic / electric transformer) from the real traffic system $\boldsymbol{\Phi}_{3}, \mathbf{I}_{3}$ can be used as an input information feedback to

\footnotetext{
${ }^{5}$ The best variant should fulfill the condition of the least actions, but it is difficult to achieve. So we only accept a good strategy.

${ }^{6}$ We only observed in detail a part of the transportation system.
} 
the original traffic model $\boldsymbol{\Phi}_{1}, \mathbf{I}_{1}$. The better input information $\boldsymbol{\Phi}_{1}, \mathbf{I}_{1}$ the better control strategy $\boldsymbol{\Phi}_{2}, \mathbf{I}_{2}$ and so on. The performance parameters [21] can be used for complex system assessment.

The illustrative example presents the links among information gates and the behavior of a real physical system. The advantage of electric / magnetic analogy is that it can easily be combined with both the real and the information components of studied system. We can order these components into more complex structures including feedbacks. Using this approach, we can model complex systems covering both their real and virtual parts.

\section{Quantum physics - information analogies}

\subsection{Quantum features}

The quantum approach is appropriate for quantum logic that combines the parallel existence of different events or processes. We can use this instrument for modelling the synergies among different parts of reality. For example, in our brain we have a lot of incomplete information obtained in different time intervals under specific conditions.

There exists a lot of paths with similar characteristics. Due to high redundancy of the neural networks we can find many parallel paths with the same measurable probabilistic characteristics (same modulus of input / output probabilities). The observer cannot distinguish among them; however, each path can carry different phase. Switching among parallel paths leads to information coding known in radioelectronics as phase modulation.

Other question asked related to quantum information system is how to use the inner quantum states. Due to quantum features we have not only two states 0 , 1 like in classical informatics but due to phase parameter we have four possible variants of q-bit: registered 0 , registered 1 , registered neither 0 nor 1 (empty set), and registered either 0 or 1 (both variants). Even if we cannot register the pure values 0 or 1 , it is still possible to distinguish between the other two variants: empty set (neither 0 nor 1 ) and both variants (either 0 or 1 ). It means that the inner states could also be used for quantum information coding.

\subsection{Quantum superposition}

Let us have $N$ variants of the available information flows $\tilde{\boldsymbol{\Phi}}_{i}$ and the information contents $\tilde{\mathbf{I}}_{i}$ assigned into the event or process ${ }^{7}$ :

$$
\tilde{\mathbf{\Phi}}_{1}, \tilde{\boldsymbol{\Phi}}_{2}, \ldots, \tilde{\boldsymbol{\Phi}}_{N}, \tilde{\mathbf{I}}_{1}, \tilde{\mathbf{I}}_{2}, \ldots, \tilde{\mathbf{I}}_{N}
$$

Due to the assumption of linearity we can sum them up to achieve the final values of the information flows and the information contents as follows:

$$
\begin{aligned}
\left|\boldsymbol{\Phi}_{1}\right\rangle & =|0,0, \ldots, 0\rangle=|0\rangle, \\
\left|\mathbf{I}_{1}\right\rangle & =|0,0, \ldots, 0\rangle=|0\rangle,
\end{aligned}
$$

\footnotetext{
${ }^{7}$ We do not distinguish in this example between the source and recipient of information
} 


$$
\begin{aligned}
\left|\mathbf{\Phi}_{2}\right\rangle & =|1,0, \ldots, 0\rangle=\left|\tilde{\mathbf{\Phi}}_{1}\right\rangle, \\
\left|\mathbf{I}_{2}\right\rangle & =|1,0, \ldots, 0\rangle=\left|\tilde{\mathbf{I}}_{1}\right\rangle, \\
\left|\boldsymbol{\Phi}_{3}\right\rangle & =|1,1, \ldots, 0\rangle=\left|\tilde{\mathbf{\Phi}}_{1}+\tilde{\mathbf{\Phi}}_{2}\right\rangle, \\
\left|\mathbf{I}_{3}\right\rangle & =|1,1, \ldots, 0\rangle=\left|\tilde{\mathbf{I}}_{1}+\tilde{\mathbf{I}}_{2}\right\rangle, \\
\ldots & \\
\left|\mathbf{\Phi}_{N}\right\rangle & =|1,1, \ldots, 1\rangle=\left|\tilde{\mathbf{\Phi}}_{1}+\tilde{\mathbf{\Phi}}_{2}+\cdots+\tilde{\mathbf{\Phi}}_{N}\right\rangle, \\
\left|\mathbf{I}_{N}\right\rangle & =|1,1, \ldots, 1\rangle=\left|\tilde{\mathbf{I}}_{1}+\tilde{\mathbf{I}}_{2}+\cdots+\tilde{\mathbf{I}}_{N}\right\rangle .
\end{aligned}
$$

The quantum information flow and the information content can be represented as a superposition of above defined $N$-variants:

$$
\begin{gathered}
\psi_{\boldsymbol{\Phi}}=\alpha_{\boldsymbol{\Phi}, 1} \cdot\left|\boldsymbol{\Phi}_{1}\right\rangle+\alpha_{\boldsymbol{\Phi}, 2} \cdot\left|\mathbf{\Phi}_{2}\right\rangle+\cdots+\alpha_{\boldsymbol{\Phi}, N} \cdot\left|\boldsymbol{\Phi}_{N}\right\rangle, \\
\psi_{\mathbf{I}}=\alpha_{\mathbf{I}, 1} \cdot\left|\mathbf{I}_{1}\right\rangle+\alpha_{\mathbf{I}, 2} \cdot\left|\mathbf{I}_{2}\right\rangle+\cdots+\alpha_{\mathbf{I}, N} \cdot\left|\mathbf{I}_{N}\right\rangle .
\end{gathered}
$$

Widely accepted criterion is the information power that requires optimization of both information flow and information content. For example, the $i, j$-th combination yields to the following information power:

$$
\left|P_{i, j}\right\rangle=\left|\boldsymbol{\Phi}_{i}, \mathbf{I}_{j}\right\rangle=\left|\left(\tilde{\boldsymbol{\Phi}}_{1}+0+\cdots+\tilde{\boldsymbol{\Phi}}_{i}+\ldots\right) \cdot\left(\tilde{\mathbf{I}}_{1}+0+\cdots+\tilde{\mathbf{I}}_{j}+\ldots\right)\right\rangle .
$$

The values $\left|\mathbf{\Phi}_{1}\right\rangle$ and $\left|\mathbf{I}_{1}\right\rangle$ need not be strictly zero. We can suppose that there exists some positive or negative information background given e.g. by common culture, education, experience, evolution. Observers do not perceive the information background behind the events, but it has an impact on the events' energy. If we imagine a negative information background it takes more energy to achieve the positive information content that orders the system. Maybe we can define the term information ecology that guarantees the positive information background.

\subsection{Illustrative example - Quantum information gyrator}

In [10] the information gyrator was firstly introduced. The gyrator is composed of information components like information nullors, information norators and information conductancies.

The information gyrator shown in Fig. 6 was used for the representation of the brain linear and nonlinear resonance phenomena [6]. A top-down expectation $\mathbf{I}_{2}$ is flowing to the short-term memory (STM) as information flow $\boldsymbol{\Phi}_{1}$. The bottom-up information flow $\boldsymbol{\Phi}_{2}$ carry the more relevant information content $\mathbf{I}_{1}$ to long-term memory (LTM).

If the output of the information gyrator is terminated by the information capacitance $^{8}$, then the input of this gyrator behaves as an information inductor.

\footnotetext{
${ }^{8}$ The information capacitance is a natural model of LTM or STM memories.
} 


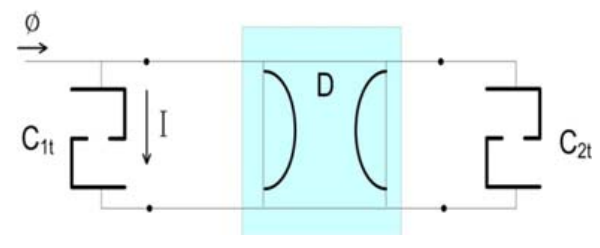

Fig. 6 Resonant connection with information gyrator [10].

If the input information flow $\boldsymbol{\Phi}$ is connected to short-term memory (capacitance $C_{1 t}$ ) then the resonant connection with the resonant frequency is obtained:

$$
f_{0}=\frac{1}{2 \cdot \pi} \cdot \frac{D^{2}}{\sqrt{C_{1 t} \cdot C_{2 t}}}
$$

where $D$ is a real constant. Consciousness seems to be the superposition of parallel quantum processes (different components of the brain connected through entanglement). Each process represents different knowledge both either historical experience or a future imagination (different scenarios of further development or fantasy).

All knowledge coded into the neural network is linked through quantum features. Rational thinking means to reduce a complexity of the possibilities' space to some tackled dimension (falling to the subset means a reduction of dimensionality). Thinking means going through the imaginable subspace of possibilities and recall the forgotten ideas due to its link to other information.

Let us imagine the quantum example of the information gyrator with a set of superposed information flows and contents:

$$
\begin{gathered}
\psi\left(\boldsymbol{\Phi}_{1}\right) \propto \alpha_{1,1} \cdot\left|\boldsymbol{\Phi}_{1,1}\right\rangle+\alpha_{1,2} \cdot\left|\mathbf{\Phi}_{1,2}\right\rangle+\cdots+\alpha_{1, N} \cdot\left|\boldsymbol{\Phi}_{1, N}\right\rangle, \\
\psi\left(\mathbf{\Phi}_{2}\right) \propto \alpha_{2,1} \cdot\left|\boldsymbol{\Phi}_{2,1}\right\rangle+\alpha_{2,2} \cdot\left|\mathbf{\Phi}_{2,2}\right\rangle+\cdots+\alpha_{2, N} \cdot\left|\boldsymbol{\Phi}_{2, N}\right\rangle, \\
\psi\left(\mathbf{I}_{1}\right) \propto \beta_{1,1} \cdot\left|\mathbf{I}_{1,1}\right\rangle+\beta_{1,2} \cdot\left|\mathbf{I}_{1,2}\right\rangle+\cdots+\beta_{1, N} \cdot\left|\mathbf{I}_{1, N}\right\rangle, \\
\psi\left(\mathbf{I}_{2}\right) \propto \beta_{2,1} \cdot\left|\mathbf{I}_{2,1}\right\rangle+\beta_{2,2} \cdot\left|\mathbf{I}_{2,2}\right\rangle+\cdots+\beta_{2, N} \cdot\left|\mathbf{I}_{2, N}\right\rangle .
\end{gathered}
$$

It means that we have at our disposal a lot of overlapping variants of information flows and contents both in STM and LTM that were created e.g. under different conditions or in different time periods. Because of the opinion and experience's diversity some information contents can be contradictory.

The gyrator input can be composed of a set of $i$-th input components $\mathbf{I}_{1, i}, \mathbf{\Phi}_{1, i}$ (a left side marked as 1 can represent a short-term memory STM) and the output of a set of $j$-th component $\mathbf{I}_{2, j}, \boldsymbol{\Phi}_{2, j}$ (a right side marked as 2 can represent a longterm memory LTM). Theoretically, the set of parallel working gyrators ${ }^{9}$ with their unique resonance frequencies is emerging. Each resonance maximizes information content assigned to the combination $\mathbf{I}_{1, i}, \boldsymbol{\Phi}_{1, i}, \mathbf{I}_{2, j}, \boldsymbol{\Phi}_{2, j}$.

In summary it yields into superposition of different resonance frequencies. From radio-electronics it is known that except for pure resonance frequencies assigned

\footnotetext{
${ }^{9}$ Their number is $i$ multiplied by $j$.
} 
to the combinations $\mathbf{I}_{1, i}, \boldsymbol{\Phi}_{1, i}, \mathbf{I}_{2, j}, \boldsymbol{\Phi}_{2, j}$ there are also created combined frequencies known as higher harmonic components. The more variants of information gyrators $\mathbf{I}_{1, i}, \boldsymbol{\Phi}_{1, i}, \mathbf{I}_{2, j}, \boldsymbol{\Phi}_{2, j}$ the more different frequencies can occur and the ability of information coding is increasing.

Considering all frequencies' variants, it is evident that a complex web of frequencies can be created. If we take each frequency as the carrier of modulated information we can bring the speculative hypothesis that our consciousness is modulated in this brain network.

The problem of high redundancy in the brain [4] is often solved through time resolution. It is expected that the fastest reply is the best one because of the shortest processing line based on accumulated knowledge. Such a situation is typical for the left- and the right-hemisphere. Both hemispheres can process the same information but due to specialization the proper one gives the fastest result.

\section{Conclusion}

In this paper a theory of a complex system was presented by using information analogy with both classical physics (electrics and magnetic circuits) and quantum physics (quantum mass parallelism and logic). The key idea is that we recognize the information flow and the information content as the main quantities in informatics as e.g. electrical current and voltage is used in electrical engineering. With respect to this idea the known mathematical instruments of electrical / magnetic circuit modelling can be applied to the modeling of complex systems.

Quantum extension of information model enables the simultaneous tackling of many superposed variants (different points of view on studied system) or in other words can work with many mutually dependent scenarios. The complex systems can be modeled as serial, parallel or feedback ordering of different quantum information gates. The achieved results can be seen as a feasibility of these analogies to tackle difficult complex system models.

Another result is the clear separation between an information source and an information recipient. The recipient sometimes cannot be able to process the transmitted information and it uses only its part. The interconnection between an information source and an information recipient could be very complex and may include non-linearity, hysteresis and also resonance relationships [10]. The presented model can consider such situations.

The achieved results shown on illustrative examples can be seen as a feasibility of these methods to tackle difficult complex systems [11,13]. The illustrative examples given above can be extended into more dimensional case. Time varying parameters could be also included. Due to high redundancy there are many variants available of solutions that can occur.

\section{Acknowledgement}

This work was supported by the Project AI \& Reasoning CZ.02.1.01/0.0/0.0/15_003/ 0000466 and the European Regional Development Fund. 


\section{References}

[1] BAR-HILlEL Y., CARNAP R. Semantic information. The British Journal for the Philosophy of Science. 1953, IV(14), pp. 147-157, doi: 10.1093/bjps/IV.14.147.

[2] BERNSTEIN D. S. Ivory ghost, IEEE Control Systems Magazine, pp. 16-17, October 2007, doi: 10.1109/MCS.2007.903688.

[3] BRAUN J. Topological analysis of networks with nullators and norators. Electronic Letters 2, 1966, pp. 427-428.

[4] FABER J., PĚKNÝ J., PIEKNÍK R., TICHÝ T., BOUCHNER P., NOVÁK M., FABER V. Simultaneous recording of electric and metabolic brain activity, Neural Network World. 2010, 20(4), pp. 539-557.

[5] FEYNMAN R., LEIGHTON R., SANDS M. Feynman lectures of physics, Addison Wesley Longman, Inc., USA, 1966.

[6] GROSSBERG S. Adaptive Resonance Theory: How a brain learns to consciously attend, learn, and recognize a changing world. Neural Networks, 37, 2012, pp. 1-47, doi: 10.1016/ j.neunet.2012.09.017.

[7] HAMILL D. C. Gyrator-Capacitor Modelling: A better Way of Understanding Magnetic Components, Applied Power Electronics Conference and Exposition, 1994. APEC '94. Conference Proceedings 1994, pp. 326-332.

[8] KAUFFMAN S. Autonomous Agents, in BARROW D. J., DAVIES P.C.W., HARPER C.L. Science and Ultimate Reality: Quantum Theory, Cosmology, and Complexity, Cambridge University Press, 2004. ISBN 9780521831130.

[9] MOOS P., MALINOVSKÝ V. Information Systems and Technologies. ČVUT, Praha 2009.

[10] MOOS P., SVÍTEK M., VOTRUBA Z., NOVÁK M. Information model of resonance phenomena in brain neural networks, Neural Network World, 2018/3, pp. 225-239, doi: 10. 14311/NNW. 2018.28.014.

[11] NOVOTNÝ S., BOUCHNER P. Advanced methodology for evaluation of driver's actual state with use of technical driving data, Proceedings of the 8th WSEAS International Conference on Systems Theory and Scientific Computation (ISTAC'08) - New Aspects of Systems Theory and Scientific Computation. Athens: World Scientific and Engineering Society Press, 2008. pp. $160-165$.

[12] PRIBYL O., HORAK T. Individual Perception of Smart City Strategies, IEEE conference, Smart Cities Symposium Prague (SCSP), 2015, pp. 1-6, doi: 10.1109/SCSP.2015.7181550.

[13] ROZHDESTVENSKIY D., BOUCHNER P. Human machine interface for future cars Changes needed, IEEE Smart Cities Symposium, Prague (SCSP) 2017, doi: 10.1109/SCSP . 2017.7973863.

[14] VLČEK J. et al. Informační výkon (Information Power), Ed. ČVUT, 2002, ISBN: 80-01 02505-5, (in Czech).

[15] SHANNON C.E. A mathematical theory of communication, Bell Syst. Tech. J., 1948, 27:379, 623.

[16] SVÍTEK M. Applying Wave Probabilistic Functions for Dynamic System Modelling, IEEE Transactions on System, Man and Cybernetics, PART C-APPLICATIONS AND REVIEWS, 2011, 5(41), pp. 674-681, doi: 10.1109/TSMCC. 2010.2093127.

[17] SVÍTEK M. Complementary variables and its application in statistics, Neural network World, 3/2007, ISSN 1210-0552, pp. 237-253.

[18] SVÍTEK M. Towards to complex system theory, Neural network World, 1/15, 2015, pp. 5-33, doi: 10.14311/NNW. 2015.25.001.

[19] SVÍTEK M. Wave Probabilistic Functions for Quantum Cybernetics, IEEE Transactions on System, Man and Cybernetics, part c - applications and reviews, 2012, 2(42), pp. 233-240.

[20] SVÍTEK M. Wave probabilistic information power, Neural Network World, 2011, 3(21), pp. 269-276, doi: 10.14311/nnw.2011.21.016. 


\section{Neural Network World 6/2018, 535-550}

[21] SVÍTEK M., NOVOVIČOVÁ J. Performance Parameters Definition and Processing, Neural Network World, 2005, 6(15), pp. 567-577.

[22] SVÍTEK M., VOTRUBA Z., MOOS P. Towards Information Circuits, Neural Network World, 2010, 2(20), pp. 241-247.

[23] SVÍTEK M., VOTRUBA Z., ZELINKA T., JIROVSKÝ V., NOVÁK M. Transport Telematics - Systemic View, 1. ed. New York: WSEAS Press, 2013. 305 p., ISBN 978-1-61804-144-9.

[24] VEDRAL V. Introduction to Quantum Information Science, Oxford University Press, 2006, http://dx.doi.org/10.1093/acprof : oso/9780199215706.001.0001.

[25] VLČEK J. Znalostní inženýrství, Monography Neural Network World, 2003, ISBN: 80903298-0-2 (in Czech).

[26] VOTRUBA Z., NOVÁK M. Alliance Approach to the Modelling of Interfaces in Complex Heterogenous Objects, Neural Network World, 2010, 5(20), pp. 609-619. 